\title{
Design Of Alias-Free Linear Phase QUADRATURE MIRROR FILTER BANKS USING EIGENVALUE-EIGENVECTOR APPROACH
}

\author{
S. K. Agrawal ${ }^{1 \#}$ and O. P. Sahu ${ }^{2}$ \\ ${ }^{1 \& 2}$ Electronics and Communication Engineering Department, \\ National Institute of Technology, Kurukshetra-136119, Haryana, India
}

\begin{abstract}
An eigenvalue-eigenvector approach for the design of two-channel linear phase quadrature mirror filter $(Q M F)$ bank is proposed. To reduce the computational complexity, finite-duration impulse response (FIR) low-pass prototype filter of the filter bank is represented by polyphase components. The design problem is formulated to optimize the filter tap weights of the low-pass prototype filter of the QMF bank to minimize an objective function, which is the weighted sum of the square error of the filter bank transfer function at the quadrature frequency, pass-band error and stop-band residual energy of the low-pass prototype filter and measure of reconstruction ripple. The objective function has been minimized by an iterative algorithm. As compared to the existing design techniques, the proposed algorithm gives better performance in terms of mean square error in stop-band, number of iterations required and stop-band edge attenuation. Design examples are presented to validate the effectiveness of the proposed method.
\end{abstract}

\section{KEYWORDS}

Filter banks, multirate signal processing, stop-band attenuation, sub-band coding.

\section{INTRODUCTION}

Finite-duration impulse response (FIR) QMF banks have been extensively used in many signal processing fields, such as automated methods for scoring tissue microarray spots [1], multi-user communication [2], image compression [3,4], trans-multiplexers used in FDM/TDM conversion [5], multi-tone modulation systems [6], ECG signal compression [7], antenna systems [8], audio industry [9], biomedical signal processing [10], sub-band coding of speech and image signals $[11,12]$ and wideband beam forming for sonar [13].

Figure 1, shows the basic structure of a two-channel quadrature mirror filter bank. In analysis section, the signal $x(n)$ is divided into two frequency sub-bands using the low-pass filter $H_{0}(z)$ and high-pass filter $H_{1}(z)$. Each sub-band signal is down-sampled by a factor of two to achieve signal compression. In the synthesis section, the sub-band signals are up-sampled by a factor of two. The output of two synthesis filters $F_{0}(z)$ and $F_{1}(z)$ are recombined to obtain the reconstructed signal $\hat{x}(n)$. The signal $\hat{x}(n)$ suffers from aliasing, magnitude and phase distortion, due to the fact that the filters $F_{0}(z), F_{1}(z), H_{0}(z)$, and $H_{1}(z)$ are not ideal [11]. These three distortions can be minimized or removed completely by appropriate selection of analysis and synthesis filters [12].

DOI : 10.14810/elelij.2014.3209 
Aliasing distortion can be canceled completely by proper choice of synthesis filters in terms of the analysis filters as:

$$
F_{0}(z)=H_{1}(-z) \text { and } F_{1}(z)=-H_{0}(-z)
$$

and the phase distortion can be eliminated using the even length linear-phase FIR filters [11]. In a QMF bank, $H_{0}(z)$ and $H_{1}(z)$ are related to each other by the mirror image symmetry condition around $\omega=\pi / 2$

$$
H_{1}(z)=H_{0}(-z)
$$

The filter bank transfer function $\left(T_{0}(z)\right)$ of such phase distortion and alias free system is given by

$$
\begin{gathered}
\hat{X}(z)=1 / 2\left[H_{0}^{2}(z)-H_{0}^{2}(-z)\right] X(z)=T_{0}(z) X(z) \\
T_{0}(z)=1 / 2\left[H_{0}^{2}(z)-H_{0}^{2}(-z)\right]
\end{gathered}
$$

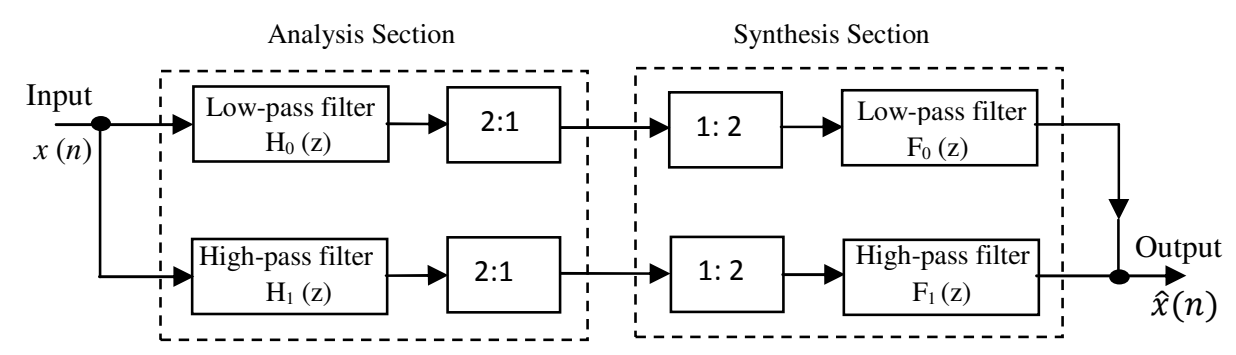

Figure 1. Basic structure of two-channel quadrature mirror filter bank

Equation (4) indicates that the overall transfer function of QMF bank depends on the filter tap weights of the low-pass analysis filter only, which is known as low-pass prototype filter. For perfect reconstruction QMF bank, magnitude distortion should also be eliminated. But due to mirror image symmetry constraint of Eq. (3), we can only minimize this distortion by using computer assistance optimization techniques [11].

Let, the prototype filter $H_{0}(z)$ is selected as even length linear -phase FIR filter, then the impulse response $h_{0}(n)$ of the prototype filter is given by inverse Z-transform of $H_{0}(z)$

$$
h_{0}(n)=h_{0}(N-n), \text { for } n=0,1,2, \ldots(N+1) / 2-1
$$

Then the Fourier transform is written as

$$
H_{0}\left(e^{j \omega}\right)=e^{-j \omega N / 2}\left|H_{0}\left(e^{j \omega}\right)\right|
$$

where $N+1$ is filter length and $e^{-j \omega N / 2}$ represents the linear phase part of prototype filter. By substituting Eq. (6) into Eq. (4), the frequency response of QMF bank is obtained as

$$
T_{0}\left(e^{j \omega}\right)=\frac{1}{2}\left(e^{-j \omega N}\right)\left[\left|H_{0}\left(e^{j \omega}\right)\right|^{2}-(-1)^{N}\left|H_{0}\left(e^{j(\omega-\pi}\right)\right|^{2}\right]
$$


Filter order $N$ must be chosen odd to avoid the severe amplitude distortion at $\omega=\pi / 2$. For perfect reconstruction QMF bank the magnitude response $\left|T_{0}\left(e^{j \omega}\right)\right|$ must be constant for all $\omega$, therefore, the condition for perfect reconstruction can be obtained as

$$
\left|T_{0}\left(e^{j \omega}\right)\right|=\left|H_{0}\left(e^{j \omega}\right)\right|^{2}+\left|H_{0}\left(e^{j(\omega-\pi)}\right)\right|^{2}=c, \quad 0 \leq \omega \leq \pi .
$$

In QMF bank, after eliminating aliasing and phase distortion completely, we can only minimize the magnitude distortion by optimizing $H_{0}(z)$ due to mirror image symmetry constraints [12]. Consequently, the design problem of QMF bank is to optimize the filter tap-weights of $H_{0}(z)$ by computer-aided optimization technique to satisfy the perfect reconstruction condition nearly. These types of filter banks are known as nearly perfect reconstruction QMF bank.

Over the past two decades many techniques have been developed to design two-channel QMF banks [14-25]. Jain and Crochiere [14] proposed an eigenvalue-eigenvector method to find the optimum prototype filter coefficients in time domain. Chen and Lee [15] presented a weighted least-squares technique in frequency domain. Several iterative methods [16-21] and genetic algorithm [22-25] applied for the design of two-channel QMF banks. Based on Johnston's [17] methods, authors in [21] have developed an efficient technique by considering polyphase components for the design of QMF bank, but the reconstruction error was high. Recently, Ghosh et al. [24] proposed an approach based on adaptive-differential-evolution algorithm and in [25] an improved particle swarm optimization (PSO) method has presented for the design of QMF banks in frequency domain.

In this paper, we present an efficient technique for design of two-channel QMF bank in which prototype low-pass filter considered in polyphase form. Inverse power method [26] has been applied for optimization of filter coefficients of low-pass prototype filter. In section 2, design of QMF bank using polyphase components and formulation of objective function are presented. In section 3, proposed algorithm for design of prototype filter is described. Design results of the proposed filter bank and comparison with existing state-of-the-art algorithms are presented in section 4.

\section{Design of Polyphase QMF bank and Formulation of ObJective FUNCTION}

Polyphase structures are very useful for implementation of filter banks [12, 27]. FIR and IIR filters can be realized with reduced computational complexity using polyphase decomposition, resulting in an efficient realization of the decimator and interpolator filter. Using the polyphase structure, an $M$-fold decimation/interpolation filter can be implemented with approximately $M$ fold reduction in the number of multiplications per unit time (MPUs) and number of additions per unit time (APUs). Type1 polyphase representation of a transfer function $H(\mathrm{z})=\sum_{n=-\infty}^{\infty} h(n) z^{-n}$, representing a digital filter, has the following $M$-component polyphase representation [11].

$$
H(z)=\sum_{k=0}^{M-1} z^{-k} E_{k}\left(z^{M}\right)
$$

where the polyphase components $E_{\mathrm{k}}(z)$ are given by 


$$
E_{k}(z)=\sum_{n=-\infty}^{\infty} e_{k}(n) z^{-n}
$$

such that

$$
e_{k}(n)=h(n M+k)
$$

$e_{k}(n)$ is the $M$-fold decimated version of $h(n+k)$. For $M=2$, the prototype filter $H_{0}(z)$ is represented using Eq. (9) as

$$
H_{0}(z)=z^{-1} E_{1}\left(z^{2}\right)+E_{0}\left(z^{2}\right)
$$

The impulse response $h_{0}(n)$ of the prototype filter $H_{0}(z)$ is symmetric: $h_{0}(n)=h_{0}(N-n)$, this also affects the polyphase components $E_{0}(z)$ and $E_{1}(z)$. The responses $e_{0}(n)$ and $e_{1}(n)$ are mirror image to each other for odd $N$ due to the symmetry of $h_{0}(n)$ [11]. The mirror image relation of $e_{0}$ $(n)$ and $e_{1}(n)$ further impact as additional saving in multiplication rate by a factor of two. Finally, the polyphase structure for a two-fold decimation/interpolation filter requires only about N/4 MPUs. The mirror image relationship between $e_{0}(n)$ and $e_{1}(n)$ is expressed as

$$
e_{0}(n)=e_{1}((N-1) / 2-n)
$$

so, $E_{1}(z)$ can be expressed in terms of as $E_{0}(z)$

$$
E_{1}(z)=z^{-(N-1) / 2} E_{0}\left(z^{-1}\right)
$$

Now, the polyphase structure described by $E_{0}(z) \& E_{1}(z)$ with noble identities in [11] can be further simplified using Eq. (14) as depicted in figure 2.

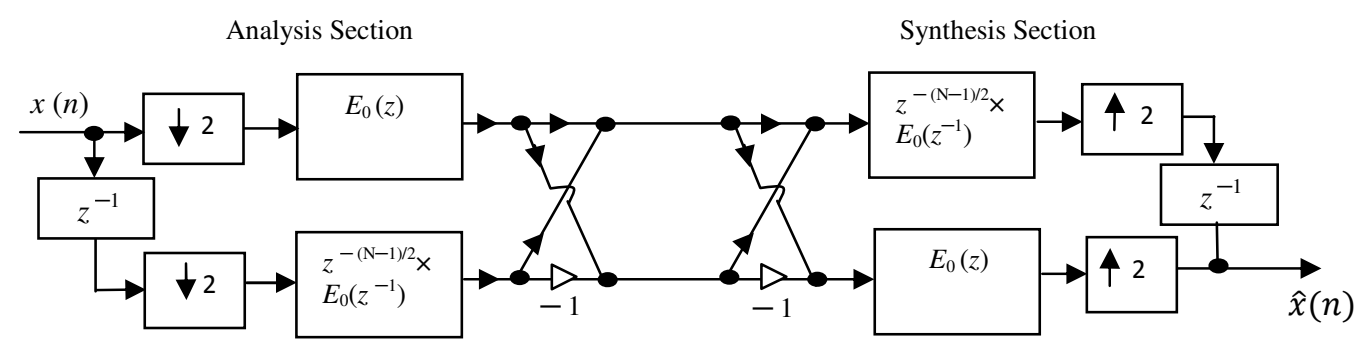

Figure 2. Two-channel QMF bank using one polyphase structure

Using Eqs.(11), (12) and (14), frequency response of low-pass analysis filter is given by

$$
\begin{aligned}
H_{0}\left(e^{j \omega}\right) & =e^{-j \omega N} \sum_{n=0}^{(N-1) / 2} e_{0}(n) e^{2 j \omega n}+\sum_{n=0}^{(N-1) / 2} e_{0}(n) e^{-2 j \omega n} \\
H_{0}\left(e^{j \omega}\right) & =\sum_{n=0}^{(N-1) / 2} e_{0}(n)\left[e^{-j \omega N} e^{2 j \omega n}+e^{-2 j \omega n}\right]
\end{aligned}
$$

The expression for $H_{0}\left(e^{j \omega}\right)$ in terms of amplitude function and linear phase component is given by

$$
H_{0}\left(e^{j \omega}\right)=e^{-j \omega N / 2}\left[\sum_{n=0}^{(N-1) / 2} 2 e_{0}(n) \cos \omega\left(2 n-\frac{N}{2}\right)\right]
$$




$$
=e^{-j \omega N / 2} \cdot \mathbf{e}_{0}^{T} \cdot \mathbf{c}(\omega)
$$

where $\mathbf{e}_{\mathbf{0}}=\left[e_{0}(0) e_{0}(1) \ldots \ldots . e_{0}(N-1) / 2\right]^{T}$ and

$\mathbf{c}(\boldsymbol{\omega})=2 \cdot\left[\cos \left(0-\frac{N}{2}\right) \omega, \cos \left(2-\frac{N}{2}\right) \omega, \ldots \ldots \ldots \cos \left(\frac{N}{2}-1\right) \omega\right]^{T}$

In this paper, we are considering the minimization of transition band error of prototype filter as well as pass-band and stop-band errors. This improves the accuracy of QMF bank that is required for real time applications. The objective function ' $K$ ' to be minimized for the QMF bank design is given as a weighted sum of four terms shown below:

$$
K=\alpha_{1} E_{p}+\alpha_{2} E_{s}+\alpha_{3} E_{t}+\alpha_{4} \cdot R R
$$

where $\alpha_{1-} \alpha_{4}$ are real constants, and $E_{\mathrm{p}}, E_{\mathrm{s}}, E_{\mathrm{t}}$, and $R R$ are the measure of pass-band error, stopband residual energy, square error of the filter bank transfer function in transition band at $\pi / 2$, and reconstruction ripple, respectively. Using Eq. (18) and (19), $E_{\mathrm{p}}, E_{\mathrm{s}}$ and $E_{\mathrm{t}}$ can be expressed as

$$
\begin{aligned}
E_{p} & =\frac{1}{\pi} \int_{0}^{\omega_{p}}\left[1-\left|H_{0}\left(e^{j \omega}\right)\right|\right]^{2} d \omega \\
& =\mathbf{e}_{0}^{T} \mathbf{P} \mathbf{e}_{\mathbf{0}}
\end{aligned}
$$

where

$$
\mathbf{P}=\frac{1}{\pi} \int_{0}^{\omega_{p}}[1-\mathbf{c}(\omega)][1-\mathbf{c}(\omega)]^{T} d \omega
$$

$E_{\mathrm{s}}$ is the stop band energy of prototype filter between $\omega_{\mathrm{s}}$ to $\pi$ is given by

where

$$
\begin{aligned}
E_{S} & =\frac{1}{\pi} \int_{\omega_{s}}^{\pi}\left|H_{0}\left(e^{j \omega}\right)\right|^{2} d \omega \\
& =\mathbf{e}_{0}^{T} \mathbf{Q} \mathbf{e}_{\mathbf{0}}
\end{aligned}
$$

$$
\mathbf{Q}=\frac{1}{\pi} \int_{\omega_{S}}^{\pi}[\mathbf{c}(\boldsymbol{\omega})][\mathbf{c}(\boldsymbol{\omega})]^{T} d \boldsymbol{\omega}
$$

$E_{\mathrm{t}}$ is the square error of $T_{0}(z)$ at quadrature frequency given by [19]

$$
\begin{aligned}
E_{t} & =\left[H_{0}(\pi / 2)-\frac{1}{\sqrt{2}} H_{0}(0)\right]^{2} \\
& =\left[\mathbf{e}_{0}^{T} \mathbf{c}(\boldsymbol{\pi} / \mathbf{2})-\frac{1}{\sqrt{2}} \mathbf{e}_{0}^{T} \mathbf{c}(\mathbf{0})\right]^{2},
\end{aligned}
$$

vector $\mathbf{c}(\boldsymbol{\omega})$ is evaluated at $\omega=\pi / 2$ and $\omega=0$ yields, vector $\mathbf{c}(\boldsymbol{\pi} / \mathbf{2})$ and $\mathbf{c}(\mathbf{0})$, respectively. $R R$ is the reconstruction ripple and expressed as [21]

$$
R R=\max _{\omega}|10 \log | T_{0}\left(e^{j \omega}\right)||-\min _{\omega}|10 \log | T_{0}\left(e^{j \omega}\right)||
$$




\section{Design of the Low-Pass Prototype Filter by Proposed ALGORITHM}

The QMF design problem is reduced to a low-pass analysis prototype filter design problem. The remaining three filters of the filter bank can be completely determined by low-pass prototype filter. In this work, the design problem is considered as an unconstrained optimization problem. The objective function $K$ is minimized by optimizing the filter tap weights of prototype filter iteratively using inverse power method based on eigenvalue-eigenvector approach. Using Eqs. (22), (25), (28) and (29), the objective function $K$ is rewritten in quadratic form

$$
\begin{gathered}
K=\alpha_{4} \cdot R R+\alpha_{3 .}\left[\mathbf{e}_{0}^{T} \mathbf{c}(\boldsymbol{\pi} / \mathbf{2})-\frac{1}{\sqrt{2}} \mathbf{e}_{0}^{T} \mathbf{c}(\mathbf{0})\right]^{2}+\alpha_{2} \cdot \mathbf{e}_{0}^{T} \mathbf{Q} \mathbf{e}_{\mathbf{0}}+\alpha_{1} \cdot \mathbf{e}_{0}^{T} \mathbf{P} \mathbf{e}_{\mathbf{0}} \\
=\alpha_{4} \cdot R R+\alpha_{3}\left[\mathbf{e}_{0}^{T} \mathbf{c}(\boldsymbol{\pi} / \mathbf{2})-\frac{1}{\sqrt{2}} \mathbf{e}_{0}^{T} \mathbf{c}(\mathbf{0})\right]^{2}+\mathbf{e}_{0}^{T} \mathbf{R} \mathbf{e}_{\mathbf{0}}
\end{gathered}
$$

where matrix $\mathbf{R}$ is

$$
\mathbf{R}=\alpha_{2} \mathbf{Q}+\alpha_{1} \mathbf{P}
$$

$K$ is further simplified as

$$
K=\alpha_{4} \cdot R R+\alpha_{3} \cdot\left[\frac{1}{2} H_{0}^{2}(0)-\sqrt{2} \cdot \mathbf{e}_{0}^{T} \mathbf{c}(\boldsymbol{\pi} / 2)\right]^{2}+\mathbf{e}_{0}^{T} \mathbf{B} \mathbf{e}_{\mathbf{0}}
$$

where matrix $\mathbf{B}$ is

$$
\mathbf{B}=\alpha_{3} . \mathbf{D}+\mathbf{R}
$$

The matrix $\mathbf{B}$ is a real, symmetric and positive definite matrix. The vector $\mathbf{e}_{0}$ which minimizes the objective function $K$ is the eigenvector corresponding to the minimum eigenvalue ' $\lambda$ ' of $\mathbf{B}$, which is calculated using inverse power method. Inverse Power method [11] is an iterative method that provides an easy way for computation of eigenvector corresponding to minimum eigenvalue ' $\lambda$ ', provided matrix $\mathbf{B}$ is nonsingular. The advantage of the inverse power method is that it converges very rapidly [26]. The detailed algorithm for designing prototype filter using proposed method is described chronologically:

Step 1: Mention even filter length $(N+1)$, band-edge frequencies: $\omega_{\mathrm{s}}$ (stop-band edge frequency) and $\omega_{\mathrm{p}} \quad$ (pass-band edge frequency), consider initial values of $\alpha_{1}, \alpha_{2}, \alpha_{3}$ and $\alpha_{4}$.

Step 2: Begin with an initial guess $\mathbf{x}_{\mathbf{i}}$ for an eigenvector of the matrix $\mathbf{B}$.

Step 3: Set a counter, $i=0$.

Step 4: Constrain initial eigenvector $\mathbf{x}_{\mathbf{i}}$ to unit norm vector as $\mathbf{e}_{\mathbf{0}(\mathbf{i})}$, and compute the objective function $K_{\mathrm{i}} \quad$ at the vector $\mathbf{e}_{\mathbf{0}(\mathbf{i})}$, by using Eq. (30).

Step 5: Obtain the inverse of matrix $\mathbf{B}$ defined by Eq (32).

Step 6: Calculate new eigenvector as $\mathbf{x}_{i+1}=\mathbf{B}^{-1} \cdot \mathbf{e}_{\mathbf{0 ( i )}}$

Step 7: Compute $\mathbf{e}_{\mathbf{0}(\mathrm{i}+1)}$ from $\mathbf{x}_{i+1}$ and calculate the objective function $K_{i+1}$, at the design vector $\mathbf{e}_{\mathbf{0}_{(\mathrm{i}+1)} \text {. If }} \quad K_{i+1} \geq K_{i}$, choose the optimum point as $\mathbf{e}_{\mathbf{0 ( \mathrm { i } )}}$, stop the procedure and go to step (9). If $K_{i+1}<K_{\mathrm{i}}$, set

$$
K_{i}=K_{i+1}, \mathbf{e}_{0(\mathbf{i})}=\mathbf{e}_{\mathbf{0}(\mathbf{i}+\mathbf{1})} \text { and } \mathbf{x}_{\mathbf{i}}=\mathbf{x}_{\mathbf{i + 1}} \text {. }
$$

Step 8: Check the condition $\mathbf{B}^{-\mathbf{1}} \cdot \mathbf{e}_{\mathbf{0}(\mathrm{i})}=\mathbf{B}^{-\mathbf{1}} \cdot \mathbf{e}_{\mathbf{0}(\mathrm{i}+1)}$, if this condition satisfied, stop the procedure and go to step (9). If not satisfied, set the new iteration number as $i=i+1$, go to step (6). 
Step 9: Compute the optimum solution as eigenvector $\mathbf{e}_{0}=\mathbf{e}_{0(\mathbf{i})}$, and minimum eigenvalue (' $\lambda$ ' $=$ $\left.1 / \lambda_{0}\right)$ of finally, calculate

matrix $\mathbf{B}$ is calculated from the relation: $\mathbf{B}^{-1} \cdot \mathbf{e}_{\mathbf{0 ( i )}}=\mathbf{B}^{-\mathbf{1}} \cdot \mathbf{e}_{\mathbf{0 ( i + 1 )}}=\lambda_{0} . \mathbf{e}_{\mathbf{0 ( i + 1 )}}$, and the optimized prototype filter coefficients $h_{0}(n)$.

\section{DeSign EXAMPLES AND DisCUSSION}

Results of typical designs are presented in this section. The performance of proposed algorithm is measured in terms of parameters: $E_{\mathrm{p}}, E_{\mathrm{s}}$, CPU time, Number of iterations (NOI), Stop-band edge attenuation $\left(A_{\mathrm{s}}\right)=-20 \log _{10}\left(H_{0}\left(\omega_{\mathrm{s}}\right)\right)$ and $R R=\max _{\omega}|10 \log | T_{0}\left(e^{j \omega}\right)||-\underset{\omega}{\min }|10 \log | T_{0}\left(e^{j \omega}\right) \|$. Matlab computer programs, which implements the proposed method are tested on Intel Core 2 Duo CPU @ 2.10 GHz, 1 GB RAM. The performance of the QMF bank designed by proposed method is compared with other available methods via Table 1 for $N+1=24$.

Example 1: Prototype filter length $(N+1)=20, \omega_{\mathrm{s}}=0.6 \pi, \omega_{\mathrm{p}}=0.4 \pi, \alpha_{1}=0.38, \alpha_{2}=0.95, \alpha_{3}=0.1$ and $\alpha_{4}=10^{-4}$, the optimized filter coefficients obtained are as follows:

$$
\begin{array}{ll}
h_{0}(19)=h_{0}(0)=-0.0043, & h_{0}(18)=h_{0}(1)=0.0141 \\
h_{0}(17)=h_{0}(2)=0.0033, & h_{0}(16)=h_{0}(3)=-0.0418 \\
h_{0}(15)=h_{0}(4)=0.0095, & h_{0}(14)=h_{0}(5)=0.0925 \\
h_{0}(13)=h_{0}(6)=-0.0554, & h_{0}(12)=h_{0}(7)=-0.1985 \\
h_{0}(11)=h_{0}(8)=0.2362, & h_{0}(10)=h_{0}(9)=0.9440
\end{array}
$$

Normalized magnitude plots of analysis filters $H_{0}(z) \& H_{1}(z)$ and reconstruction ripple (in $\mathrm{dB}$ ) of QMF bank are plotted in fig. 3a and fig.3b, respectively. The parameters obtained are $E_{\mathrm{p}}=$ $9.71 \times 10^{-7}, E_{\mathrm{s}}=7.15 \times 10^{-6}, A_{\mathrm{s}}=28.67 \mathrm{~dB}$, CPU-time $=0.0032 \mathrm{sec} ., R R($ in $\mathrm{dB})=0.039$ and $\mathrm{NOI}=$ 02.

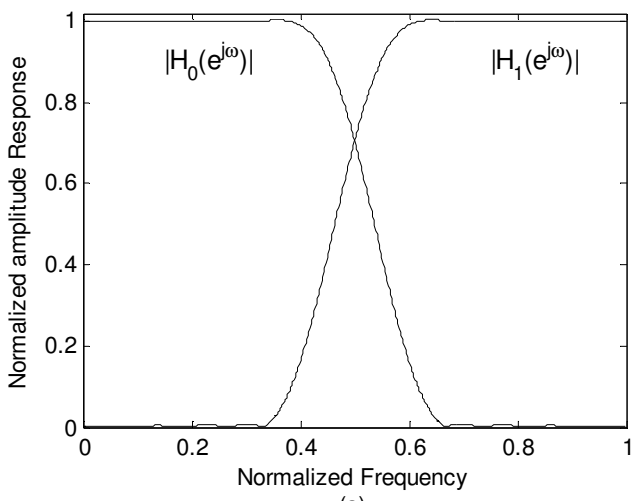

(a)

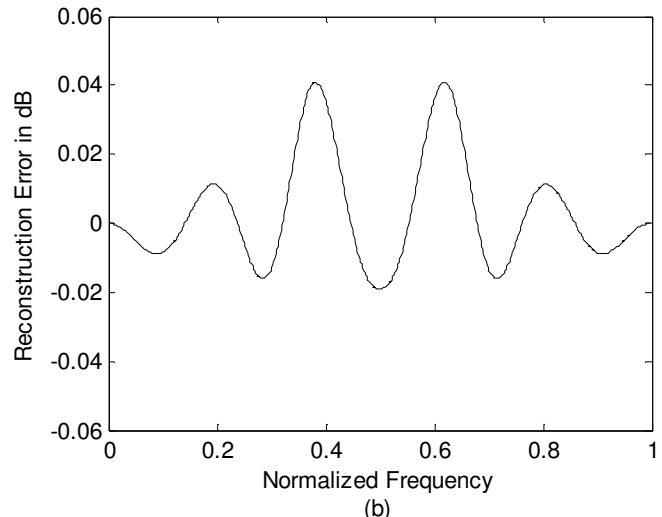

(b)

Fig. 3 (a) Magnitude response of analysis filters for $N+1=20$. (b) Reconstruction ripple in $\mathrm{dB}$.

Example 2: Prototype filter length $(N+1)=24, \omega_{\mathrm{s}}=0.6 \pi, \omega_{\mathrm{p}}=0.4 \pi, \alpha_{1}=0.56, \alpha_{2}=0.95, \alpha_{3}=0.1$ and $\alpha_{4}=10^{-4}$, the optimized filter coefficients obtained are as follows:

$$
\begin{array}{ll}
h_{0}(23)=h_{0}(0)=-0.002179, & h_{0}(22)=h_{0}(1)=0.006418, \\
h_{0}(21)=h_{0}(2)=0.003350, & h_{0}(20)=h_{0}(3)=-0.02097, \\
h_{0}(19)=h_{0}(4)=-0.00019, & h_{0}(18)=h_{0}(5)=0.04854,
\end{array}
$$


Electrical and Electronics Engineering: An International Journal (ELELIJ) Vol 3, No 2, May 2014

$$
\begin{array}{ll}
h_{0}(17)=h_{0}(6)=-0.01557, & h_{0}(16)=h_{0}(7)=-0.097285 \\
h_{0}(15)=h_{0}(8)=0.06268, & h_{0}(14)=h_{0}(9)=0.19904 \\
h_{0}(13)=h_{0}(10)=0.24299, & h_{0}(12)=h_{0}(11)=-0.94066
\end{array}
$$

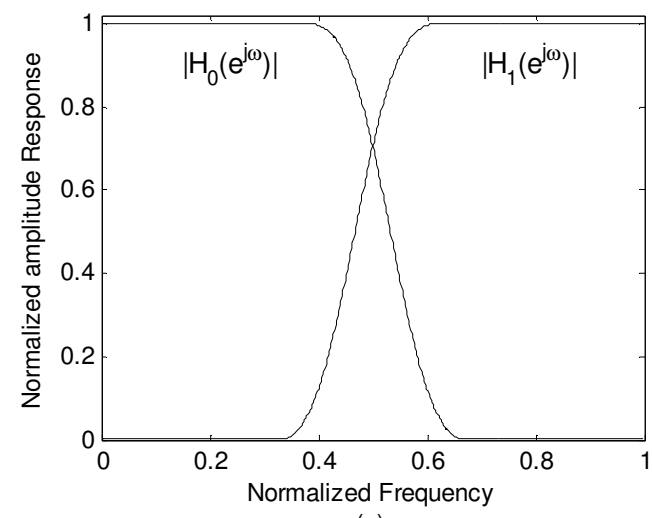

(a)

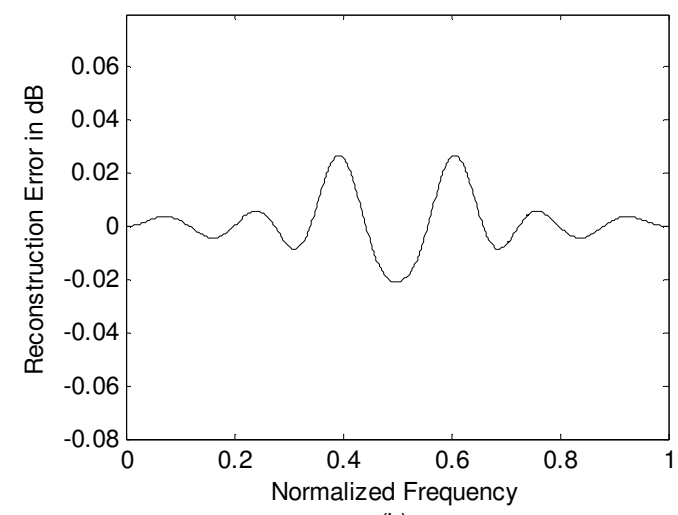

(b)

Fig. 4 (a) Magnitude response of analysis filters for $N+1=24$. (b) Reconstruction ripple in $\mathrm{dB}$. Normalized magnitude plots of analysis filters $H_{0}(z) \& H_{1}(z)$ and reconstruction ripple (in dB) of QMF bank are plotted in fig. $4 \mathrm{a}$ and fig. $4 \mathrm{~b}$, respectively. The parameters obtained are $E_{\mathrm{p}}=1.919 \times 10^{-7}, E_{\mathrm{s}}=$ $9.707 \times 10^{-7}, A_{\mathrm{s}}=31.52 \mathrm{~dB}$, CPU-time $=0.0037 \mathrm{sec}$., $R R($ in $\mathrm{dB})=0.027$ and $\mathrm{NOI}=02$.

Table 1: Performance comparison of proposed inverse power method with other methods $\left(\omega_{\mathrm{s}}=0.6 \pi, \omega_{\mathrm{p}}=\right.$

\begin{tabular}{lccccc}
\hline Name of the Method & NOI & $E_{\mathrm{p}}$ & $A_{5}(\mathrm{~dB})$ & $R R(\mathrm{~dB}$ & $E_{5}$ \\
& & & & & \\
\hline Steepest-Descent [21] & 76 & $1.32 \times 10^{-7}$ & 25 & 0.031 & $7.06 \times 10^{-5}$ \\
Levenberg-Marq. [20] & 21 & $7.86 \times 10^{-8}$ & 28.31 & 0.017 & $1.74 \times 10^{-5}$ \\
Neural Networks [24] & 09 & --- & 22.05 & 0.043 & - \\
PSO [23] & 122 & $1.84 \times 10^{-7}$ & 22.78 & 0.019 & $7.99 \times 10^{-5}$ \\
Marquardt [19] & 109 & $9.23 \times 10^{-8}$ & 23.03 & 0.025 & $8.45 \times 10^{-5}$ \\
MJADE_pBX algo. [25] & $-\ldots--$ & $9.14 \times 10^{-7}$ & 20.11 & 0.030 & $1.30 \times 10^{-4}$ \\
Proposed Method & $\mathbf{0 2}$ & $\mathbf{1 . 9 1 9 \times 1 0 ^ { - 7 }}$ & $\mathbf{3 1 . 5 2}$ & $\mathbf{0 . 0 2 7}$ & $\mathbf{9 . 7 0 7 \times 1 0 ^ { - 7 }}$ \\
& & & & & \\
\hline
\end{tabular}

From the comparison table, it is seen that the proposed polyphase filter approach with inverse power method required less computational efforts than the other existing methods. The proposed method shows improved performance in terms of number of iteration (NOI) required, stop-band edge attenuation $\left(A_{s}\right)$ and stop band error $\left(E_{\mathrm{s}}\right)$ than all other methods. 
Electrical and Electronics Engineering: An International Journal (ELELIJ) Vol 3, No 2, May 2014

\section{Conclusions}

A design method based on polyphase filters and inverse power method was proposed for designing of two-channel QMF bank. Due to the introduction of polyphase filters, the QMF bank became very computationally efficient. The advantage of the inverse power optimization method is that it converges very rapidly therefore, number of iterations (NOI) required very less. By simulation results, it was observed that proposed method seems as an efficient alternate approach for designing high-order QMF banks. This approach may be extended for design of M-channel QMF for further work.

\section{REFERENCES}

[1] Trang Kim Le.: Automated method for scoring breast tissue microarray spots using Quadrature mirror filters and support vector machines" 15th International conference on Information (FUSION), 1868 -1875 (2012).

[2] Sablatash, M.: Designs and architectures of filter bank trees for spectrally efficient multi-user communications: review, modifications and extensions of wavelet packet filter bank trees. Signal, Image and Video Processing (SIViP), Vol. 5, No. 1, 09-37 (2008).

[3] Xia, T., Jiang, Q.: Optimal multifilter banks: Design related symmetric extension transform and application to image compression. IEEE Trans. Signal Process., 47 (7), 1878-1889 (1995).

[4] Smith, M. J. T., Eddins, S. L.: Analysis / synthesis techniques for sub-band image coding. IEEE Trans. Acoust. Speech Signal Process., ASSP-38(8), 1446-1456 (1990).

[5] Bellanger, M. G., Daguet, J. L.: TDM-FDM trans-multiplexer: Digital polyphase and FFT. IEEE Trans. Commun. 22 (9), 1199-1204 (1974).

[6] Vetterli, M.: Multidimensional sub-band coding: Some theory and algorithms. Signal Process. 6, 97$112(1984)$

[7] Afonso, V. X., Tompkins, W. J., Nguyen, T. Q., Luo, S.: ECG beat detecting using filter banks. IEEE Trans. Biomed. Engg., 46(2), 192-202 (1999).

[8] Chandran, S.: A novel scheme for a sub-band adaptive beam forming array implementation using quadrature mirror filter banks. Electron. Lett., 39(12), 891-892 (2003).

[9] Painter, T., Spanias, A. : Perceptual coding of digital audio. Proc. IEEE, 88(4), 451-513 (2000).

[10] Kumar, A., Singh, G. K., Anand, R. S.: An improved method for the designing quadrature mirror filter banks via unconstrained optimization. J. Math. Model. Algorithm 9(1), 99-111 (2010).

[11] Vaidyanathan, P. P.: Multirate systems and filter banks. Prentice Hall, Englewood Cliffs, NJ (1993).

[12] Vaidyanathan, P. P.: Multirate digital filters, filter banks, polyphase networks and applications: A tutorial. Proc. IEEE 78 (1), 56-93 (1990).

[13] H. Charafeddine, V. Groza, Wideband adaptive LMS beamforming using QMF sub-band decomposition for sonar, in: 8th IEEE Int. Symp. on Applied Computational Intelligence and Informatics, Romania, 2013, pp. 431-436.

[14] Jain, V. K., Crochiere, R. E.: Quadrature mirror filter design in time domain. IEEE Trans. Acoust. Speech Signal Process. ASSP-32 (4), 353-361 (1984).

[15] Chen, C.K., Lee, J.H.: Design of quadrature mirror filters with linear phase in the frequency domain. IEEE Trans. Circuits Syst. 39 (9), 593-605 (1992).

[16] Xu, H., Lu, W. S., Antoniou, A.: An improved method for the design of FIR quadrature mirror image filter banks. IEEE Trans. Signal Process.46 (6), 1275-1281 (1998).

[17] J. D. Johnston, "A Filter Family Designed for Use in Quadrature Mirror Filter Banks," Proc. IEEE, Int. Conf. AS SP, pp. 292 - 294, April (1980).

[18] Sahu, O. P., Soni, M. K., Talwar, I. M.: Marquardt optimization method to design two channel quadrature mirror filter banks. Digital Signal Process.16(6), 870-879 (2006). 
[19] Kumar, A., Singh, G. K., Anand, R. S.: An improved method for the design of quadrature mirror filter bank using the Levenberg-Marquardt Optimization. Signal, Image and Video Processing (SIViP) DOI: 10.1007/s11760-011-0209-9 (2011).

[20] Sahu, O. P., Soni, M. K., Talwar, I. M.: Designing quadrature mirror filter banks using steepest descent method. Jorunal of Circuits Systems and Computers, Vol. 15, No. 2, 29-42 (2006).

[21] Gupta, A., Agrawal, S. K. : Designing of Two Channel Polyphase Quadrature Mirror Filter Bank using Power Optimization Method. International Conference on Computer \& Communication Technology (ICCCT), (2011).

[22] J. Upendar, C. P. Gupta, G. K. Singh, Designing of two channel quadrature mirror filter bank using Partical Swarm Optimization, Digital Signal Processing 20 (10) (2010) 304-313.

[23] Yue-Dar Jou, Design of two-channel linear-phase quadrature mirror filter banks based on neral networks,Signal Processing 87 (2007) 1031-1044.

[24] Pradipta Ghosh, Swagatam Das and Hamim Zafar, "Adaptive-differential-evolution-based Design of two - channel quadrature mirror filter banks for sub-band coding and data transmission," IEEE Trans. On Systems, Man, And Cybernetics-Part C: Applications And Reviews, Vol. 42, No. 6,pp. 1613-1623, November 2012.

[25] Rafi, S. M., Kumar, A., Singh, G. K.: An improved particle swarm optimization method for multirate filter bank design. Journal of the Franklin Institute, 350, 757-759 (2013).

[26] Bronson R. and Costa G. B. : Linear algebra an introduction . Elsevier (2009).

[27] S. K. Agrawal and O. P. Sahu, "Two-Channel Quadrature Mirror Filter Bank: An Overview," ISRN Signal Processing (Hindawi), vol. 2013, Article ID 815619, 10 pages, 2013. doi:10.1155/2013/815619.

\section{Authors}

Surendra Kr. Agrawal, He has received B.E. and M. E. degrees in 1998 and 2007, respectively. He is pursuing his Ph. D. degree from National Institute of Technology (NIT), Kurukshetra, India. His research interests are in the areas of multirate signal processing and digital communication.

O. P. Sahu, He is Professor at Department of Electronics and Communication Engineering, National Institute of Technology, Kurukshetra, India. He has more than 75 papers in his credit in various national and international conferences and journals. His research interests and specialization areas include signals and systems, digital signal processing, communication systems and fuzzy systems. 